\title{
Neue Verfassungsstrukturen in Georgien
}

\author{
Von Wolfgang Gaul
}

"Die Unterzeichnung der Verfassung wird man nicht verhindern können", sagte Eduard Schewardnadse am 29. August 1995 nach einem Attentatsversuch auf ihn, das die für diesen Tag vorgesehene Unterzeichnung der neuen georgischen Verfassung nur um wenige Wochen verzögern konnte. Das mag in Erinnerung rufen, unter welchen Umständen und Vorzeichen die Verfassung in der Kaukasusrepublik entstand.

\section{A. Die Verfassungsgebung \\ I. Die Verfassung von 1921}

In Georgien wurde schon einmal, im Februar 1921, eine eigenständige Verfassung verabschiedet. An dieses Dokument konnte man anknüpfen, selbst wenn 1921, bereits vier Tage später, die GSSR, die "Georgische Sowjetische Sozialistische Republik", proklamiert wurde: Die Rote Armee war in Georgien einmarschiert und hatte die knapp dreijährige Unabhängigkeit des Landes beendet.

Die Verfassung von 1921 galt zur damaligen Zeit als eine der fortschrittlichsten und beinhaltete viele sozialdemokratische Elemente. Sie enthielt beispielsweise umfangreiche sozial-ökonomische Rechte (Kapitel XIII) und ein Kapitel mit Rechten nationaler Minderheiten (Kapitel XVI). Die staatsorganisationsrechtlichen Passagen in der Verfassung von 1921 waren dagegen kurz gehalten. Sie entwarfen eine parlamentarische Republik: Das Parlament konnte nicht aufgelöst werden. Es wählte den Vorsitzenden der Regierung auf ein Jahr. Eine Wiederwahl war nur einmal zulässig (Art. 67 GV von 1921). Der Regierungschef ernannte sodann sein Kabinett, ohne auf eine Zustimmung des Parlamentes angewiesen zu sein (Art. 68 GV von 1921). 


\section{Die "Nationalversammlung"}

Genau 71 Jahre später ließ man die Prinzipien dieser Verfassung wieder aufleben. Der "Militärrat" ${ }^{1}$, der den Präsidenten Gamsachurdia gestürzt hatte, verkündete am 21. Februar 1992 den "Vorrang (...) der Verfassung der Demokratischen Republik Georgien vom 21. Februar 1921 sowie die Wiederherstellung der Geltung ihrer Bestimmungen unter Berücksichtigung der gegenwärtigen Realitäten und ohne Änderung der gegenwärtigen Grenzen und der national-staatlichen Verfassung". Diese Generalklausel wurde gewählt, weil der Wortlaut der alten Verfassung nicht mehr komplett auf die neue Republik Georgien zutraf. Zur Legitimation berief man eine "Nationalversammlung" zwecks Akklamation ein, in der viele namhafte Personen des öffentlichen Lebens auftraten, allerdings keine Anhänger der Opposition zu finden waren. Überdies beschloß der Militärrat das Fortbestehen der geltenden Gesetzgebung, sofern diese nicht mit den Prinzipien der Verfassung der Republik Georgien kollidierte.

Um den aus Moskau im März 1992 zurückgekehrten, ehemaligen sowjetischen Außenminister Eduard Schewardnadse bereichert, mutierte der "Militärrat" unter dem Vorsitz des "Zivilisten" Schewardnadse zum "Staatsrat"" ${ }^{2}$ Dieser bestätigte die "Geltung der Gesetzgebung der Republik Georgien in Zusammenhang mit der Wiederherstellung der Geltung der georgischen Verfassung von 1921".

\section{Das "Gesetz über die staatliche Macht"}

Dennoch war man nach dem Putsch gegen Gamsachurdia bestrebt, die Staatsstrukturen demokratischer zu legitimieren: Die Prinzipien der Verfassung von 1921 wurden durch das "Gesetz über die staatliche Macht" vom 10. November 1992 abgelöst, welches das neugewählte georgische Parlament gleich bei seiner ersten Zusammenkunft verabschiedete. Darin wurden per Gesetz die "Befugnisse der höchsten Organe des Staates, die Regeln für deren Aufbau und Tätigkeit bis zum Inkrafttreten der neuen Verfassung" bestimmt. ${ }^{4}$

Er wurde von Tengis Kitowani, dem früheren Kommandanten der Nationalgarde und Djaba Iosseliani, dem Anführer der paramilitärischen Vereinigung ,Mchedrioni“ gebildet.

2 Mitglieder des Präsidiums waren Tengis Kitowani, Djaba Iosseliani sowie der ehemalige Premierminister Tengis Sigua und Eduard Schewardnadse.

3 Beschluß des Staatsrates vom 17. Juni 1992.

4

Vgl. Präambel des Gesetzes. 
Ähnlich der Verfassung von 1921 kam auch hier dem Parlament eine Schlüsselposition zu, denn dieses wählte das Staatsoberhaupt ${ }^{5}$. Weiterhin wurde der Posten des Ministerpräsidenten geschaffen, der auf Vorschlag des Staatsoberhauptes vom Parlament ernannt wurde (Art. 8 Abs. 1 des Gesetzes). Die Ministerposten wurden wie folgt besetzt: Das Staatsoberhaupt ernannte auf Vorschlag des Ministerpräsidenten die Minister oder entließ sie. Dazu bedurfte er jedoch der Bestätigung des Parlamentes (Art. 17 Abs. 7 des Gesetzes). Auch die Struktur des Kabinetts wurde vom Parlament bestätigt (Art. 26 des Gesetzes).

Das Parlament durfte jedoch die Vollmachten des Staatsoberhauptes aufheben und über den Rücktritt des Ministerpräsidenten, der Mitglieder des Ministerkabinetts sowie über die Vertrauensfrage dieser Amtsinhaber entscheiden (Art. 8 Abs. 2 des Gesetzes). Das Parlament konnte zudem Akte des Staatsoberhauptes, des Ministerkabinetts, sowie der örtlichen Legislativ- und Verwaltungsorgane aufheben, falls sie gegen Gesetze der Republik Georgien verstieBen (Art. 7 Abs. 7 des Gesetzes ).

Ein Aufbau mit verschiedenen checks and balances also, der sich staatspolitisch durchdacht anhörte, allerdings einen entscheidenden Haken hatte: Die Ämter des Parlamentsvorsitzenden und des Staatsoberhauptes fielen de jure zusammen. De facto konzentrierte sich somit ein Großteil der Macht in der Person Eduard Schewardnadses. Annähernd drei Jahre - bis zur Verabschiedung der neuen georgischen Verfassung - war er sowohl Vorsitzender des Parlamentes, als auch Staatsoberhaupt mit exekutiven Funktionen.

\section{Die Verfassungskommission}

Die Verfassungskommission, welche die Aufgabe hatte, eine neue Verfassung auf der Grundlage der Verfassung von 1921 vorzubereiten, wurde am 19. Februar 1993 ins Leben gerufen. Ihr gehörten 118 Mitglieder an. Es wurden so viele Mitglieder integriert, "damit niemand auf der Straße blieb." Man wollte mögliche Widersacher von Beginn an mit einbeziehen.

Vorsitzender der Verfassungskommission wurde Eduard Schewardnadse, Staatsoberhaupt und Vorsitzender des Parlaments. Zu seinen Stellvertretern wurden Wachtang Chmaladse, Physiker und Vorsitzender der gemäßigten Oppositionspartei "Republikaner" und Giwi Intskirweli, den Dekan der juristischen Fakultät der staatlichen Universität (einen Posten, den dieser bereits vor 50 Jahren (!) innehatte) gewählt. Sekretär der Verfassungskommis-

5

Man scheute seinerzeit davor zurück, den Titel "Präsident" zu vergeben. Dieser war durch Gamsachurdia desavouiert worden.

$6 \quad$ Ugrechelidse, persönliches Interview am 7. August 1996. 
sion wurde Avtandil Demetraschwili, seinerzeit Professor für Verfassungsrecht an der Staatsuniversität und mittlerweile Präsident des georgischen Verfassungsgerichts.

Daraufhin begann ein zweieinhalb Jahre dauerndes Feilschen über Vorschläge und Formulierungen. Der ursprüngliche Plan, nur die Verfassung von 1921 zu redigieren, erwies sich als unpraktikabel und wurde von allen Mitgliedern der Verfassungskommission bald fallengelassen.

\section{Die Entwürfe}

\subsection{Der Chmaladse-Entwurf}

Der im wesentlichen durch ein Team um Chmaladse formulierte Entwurf wurde im Namen der Partei "Republikaner" eingebracht. Der umfangreiche Entwurf (fast 200 Artikel) war hinsichtlich der Machtstrukturen an die bundesdeutsche Verfassung angelehnt und repräsentierte ein parlamentarisches Modell. Dies war der einzige Entwurf, in dem der Präsident vom Parlament in indirekter Wahl gewählt wurde (Art. 98).

Nach dem Entwurf konnte der Präsident dem Parlament einen Kandidaten für das Amt des Premierministers vorschlagen. Lehnte das Parlament den Kandidaten ab, konnte es eigenständig einen Premierminister wählen (Art. 120 Abs. 1) und diesen gegebenenfalls später durch ein Mißtrauensvotum zu Fall bringen (Art. 128 und 129).

\subsection{Der Sekretariatsentwurf}

Der Sekretariatsentwurf stand unter der Federführung Demetraschwilis und war anfänglich als Revision der Verfassung von 1921 angelegt. Nach und nach wurden jedoch Veränderungen zugunsten eines semi-präsidialen Modells eingefügt, welches dem Aufbau der 5. Französischen Republik ähnelt. Im Gegensatz zur französischen Verfassung, nach der der Präsident den Premierminister ernennt (vgl. Art. 8 Abs. 1 Satz 1), konnte er den Premierminister im Sekretariatsentwurf nur nominieren. Wurde er vom Parlament nicht gewählt, wurde das Verfahren wiederholt (Art. 64 Abs. 5 Sekretariatsentwurf).

\subsection{Der Studentenentwurf}

Der Studentenentwurf wurde auf Initiative Demetraschwilis von jungen Studenten der Fakultät für Völkerrecht und internationale Beziehungen der Staatsuniversität erarbeitet und stellte den juristisch ausgefeiltesten Verfassungsentwurf dar. Dabei überzeugte er durch deutliche Abgrenzungen der Kompetenzen der einzelnen Machtorgane. Er verkörperte trotz eines Premierministers das am stärksten präsidial orientierte Modell der drei 
Entwürfe, kombinierte jedoch Instrumente verschiedener Verfassungen miteinander. Die Ernennung des Premierministers ist fast identisch mit dem dreifachen Kandidatenvorschlag des russischen Präsidenten an die Duma.

Ähnlich der französischen Verfassung (vgl. Art. 49 Abs. 3) konnte der Premierminister einen besonders wichtigen Gesetzesentwurf mit der Vertrauensfrage verbinden (Art. 66 Abs. 1). Lehnte das Parlament diesen ab, so konnte der Präsident ein Referendum anberaumen. Wurde dieses Referendum positiv entschieden, so konnte der Präsident das Parlament auflösen (Art. 66 Abs. 2). Auch hier konnte das Parlament den Premierminister durch ein Mißtrauensvotum stürzen (Art. 65 Abs. 1)

\section{Die internationale Beratung}

$\mathrm{Zu}$ diesem Zeitpunkt setzte eine intensive Beratung durch ausländische Experten ein, die im wesentlichen durch drei Gremien koordiniert wurde:

\subsection{Der Europarat}

Die Kommission für Demokratie und Recht des Europarates, die sogenannte VenedigKommission, hat den Verfassungsentwurf Georgiens zweimal ausführlich schriftlich kommentiert. Das betraf allerdings nur den (überarbeiteten) Sekretariatsentwurf. Bearbeiter aus verschiedenen Ländern kommentierten dabei jeweils die einzelnen Kapitel des Verfassungsentwurfs. ${ }^{7}$ Demetraschwili selbst vertrat Georgien in dieser Kommission.

\subsection{Die OSZE}

Die OSZE hat im Warschauer Büro für demokratische Institutionen und Menschenrechte (ODIRH) an der Formulierung des Grundrechtskatalogs und an der Ausarbeitung des Verfassungsgerichtsgesetzes mitgewirkt. Hier wurden die Gesetzestexte in einer kleinen Gruppe redigiert. ${ }^{8}$

\subsection{Die Sorows-Stiftung}

Das "Constitutional Legislative Policy Institute" (COLPI) in Budapest hat die Verfassungsgebung in Georgien ausführlich betreut und begleitet. In Chicago organisierte COLPI im

7

8

Im wesentlichen durch den Richter des Obersten Gerichtes Juri Tabutsadse, Chmaladse, Dr. Gia Meparischwili,, Sasa Namoradse (COLPI), und Prof. Dr. Herman Schwarz (American University in Washington D.C.) sowie Bobby Fischer (ODIHR). 
Zusammenwirken mit dem "Center for Study of Constitutionalism in Eastern Europe" der University of Chicago eine einwöchige Konferenz. Die maßgeblichen georgischen Akteure ${ }^{9}$ kamen auf neutralem Boden zusammen, um sich "die Hände dreckig zu machen" ${ }^{10}$. Obwohl dem Parlament selbstverständlich letztlich die Entscheidung über die Annahme einer neuen Verfassung vorbehalten blieb, sollten sich die georgischen Juristen und Politiker hier auf eine Kompromißfassung einigen. Die ausländischen Experten übernahmen dabei die Funktion von Koordinatoren und Beratern. ${ }^{11}$ Die Analyse der Tonbandaufzeichnungen belegt, daß sie die Diskussion mit treffenden Argumenten wesentlich voranbrachten, ohne den Georgiern Entscheidungen abzunehmen. Nach gemeinsamen Anstrengungen entstand der sogenannte "Chicago-Entwurf", der ein ausgeklügeltes System von gegenseitiger Kontrolle im Staatsgefüge vorsieht, was im Zweifel dem Parlament die letzte Entscheidung zubilligt. Insbesondere die Machtverteilung zwischen Präsidenten und Parlament wurde in Chicago ausgiebig diskutiert.

Für eine stärkere präsidiale Macht wurde - kurz zusammengefaßt - insbesondere vorgebracht, Georgien befinde sich in einer Übergangsperiode. Bedingt durch die Handlungsunfähigkeit der Staatsgewalt, benötige man Durchgriffskompetenzen, die sich in einer starken Hand vereinigten. Im Volk sei ein anderes Modell nicht durchsetzbar - es verlange nach einem starken Führer und sei nicht bereit, eine parlamentarische Republik zu tragen. In einer parlamentarischen Demokratie gäbe es oft keine klaren Mehrheiten - es müßten Koalitionen gebildet werden, die den Staat in der Durchsetzung von Reformen schwächten. Die Parteien in Georgien seien konturlos. Das Parlament solle deshalb keine große Verantwortung tragen. Schließlich sei Schewardnadse ein Garant für Stabilität. Das Präsidentenamt ohne Ansehen seiner Person zu formulieren, sei realitätsfern. Darüber hinaus nutze das Argument, daß eine Verfassung nicht für 10 Jahre, sondern für Jahrhunderte geschaffen werde, nichts, da 10 Jahre in einer Übergangsphase viel Zeit bedeuten. Danach könne die Verfassung unter Umständen auch wieder geändert werden.

Demgegenüber wurde - ebenfalls knapp zusammengefaßt - für eine stärkere parlamentarische Macht vorgebracht, das präsidiale System funktioniere zwar in Staaten mit langjähriger demokratischer Tradition wie z.B. den USA. Fehle diese, wie oft in Lateinamerika, so stünden sich Parlament und Präsident als Gegenspieler blockiert gegenüber, eine Pattsitua-

9

Teilnehmer waren Chmaladse, Intskirweli, Demetraschwili, der Vorsitzende des Obersten Gerichts Mindia Ugrechelidse, der ehemalige Justizminister Djoni Chezuriani, Prorektor der Universität Lewan Aleksidse, Tabutsadse sowie die Politiker Otar Melkadse, Gia Djordjoliani und Irakli Schengelaia.

10 So Chmaladse, persönliches Interview vom 4. Juli 1996.

11

Maßgebliche ausländische Ratgeber waren die Professoren Alexander Blankenagel (Humboldt Universität Berlin), Steven Holmes (University of Chicago), Lawrence Lessig, (University of Chicago), Andras Sajo (Central European University in Budapest), Herman Schwarz (American University in Washington D.C.), John Whyte, (Queen's University in Kingston, Canada), sowie Duc Trang (COLPI). 
tion, die in diesen Ländern gerade beim Aufbau demokratischer Strukturen oft nur durch einen Umsturz aufgelöst worden sei. Komme der Präsident, z.B. gegenüber Rußland, unter Druck, so könne er sich auf eine Entscheidung des Parlaments berufen, die ihm die Hände binde. Schewardnadse selbst bestand darauf, die Verfassung nicht auf ihn zuzuschneidern, da sie lange Bestand haben müsse.

Letztlich entschied sich die georgische Delegation in Chicago im Gegensatz zu den Modellen anderer GUS-Staaten für eine deutliche Begrenzung präsidialer Macht, was sich in folgenden Passagen des "Chicago-Entwurfes" widerspiegelt:

Der direkt gewählte Präsident (Art. 72 Abs. 1) ernannte den Premierminister (Art. 82). Dieser nominierte das Kabinett, das en bloc vom Parlament bestätigt werden mußte. Bei Ablehnung wiederholte sich der Vorgang. Im dritten Anlauf konnte das Parlament selbst einen Kandidaten bestimmen.

Der Premierminister konnte über eine konstruktives Mißtrauensvotum zu Fall gebracht werden (Art. 85 Abs. 2). Entließ er mehr als ein Drittel seiner Minister, so mußte er dem Parlament die Vertrauensfrage stellen (Art. 84 Abs. 1).

Der Präsident hatte im Gesetzgebungsverfahren kein eigentliches Vetorecht. Er konnte aber die Gesetzesvorlage mit Vorschlägen an das Parlament zurückverweisen. Das Parlament konnte den Vorschlägen mit derselben Stimmenzahl wie bei der ursprünglichen Annahme zustimmen (Art. 66 Abs. 5).

\section{Die entscheidende Phase}

Kaum waren die Teilnehmer der Konferenz mit dem neu erarbeiteten "Chicago-Entwurf" nach Georgien zurückgekehrt, erklärte der Justizminister diesen zwar für wissenschaftlich hervorragend konzipiert, jedoch untauglich in Anbetracht der tatsächlichen Situation in Georgien. Diese würde eine stärkere präsidiale Macht zwingend erfordern. Unterstützt wurde er dabei von einigen derer, die den "Chicago-Entwurf" noch einige Wochen zuvor mitgetragen hatten.

Als entscheidender Nachteil stellte sich die Tatsache heraus, daß Justizminister Tedo Ninidse trotz einer Einladung nicht an der Chicagoer Konferenz teilnahm. Im Nachhinein drängt sich sogar der Verdacht auf, daß Ninidse bewußt nicht an der Konferenz in Chicago teilgenommen hat, bzw. von Schewardnadse dazu angehalten wurde ${ }^{12}$. Bis dato hatte sich

12

Ninidse konnte sich später nicht einmal mehr an die Einladung erinnern; persönliches Interview vom 3. August 1996. 
Schewardnadse nur wenig in die Verfassungsgebung eingeschaltet. Die Verfassungsgebung wurde daraufhin von einer kleinen Gruppe um Schewardnadse und Ninidse koordiniert. Während man sich bemühte, die juristisch prägnanten Formulierungen unangetastet zu lassen, so daß sich viele Ergebnisse aus Chicago in der heute geltenden Verfassung wiederfinden, begann man den Entwurf grundsätzlich zugunsten einer stärkeren präsidialen Macht umzustrukturieren. Dabei wurden die bisherigen Ergebnisse hinsichtlich der Machtverteilung auf den Kopf gestellt, was sich bis in eine Einschränkung des Grundrechtskatalogs erstreckte.

So wirft die "Vereinigung Junger Georgischer Juristen" (GYLA), ein Zusammenschluß qualifizierter und reformorientierter Juristen, den Verantwortlichen vor, einen unpraktikablen und theoretisch inkorrekten Vorschlag unterbreitet zu haben. Bei seiner Erstellung seien politische Parteien und Spezialisten aus der Verfassungsgebung gezielt ausgegrenzt worden.

Eine ausgleichende Rolle spielte in der letzten Phase vor allem das Parlament, in der Chmaladses "Republikaner" die für die Verfassungsänderungen notwendige Zweidrittelmehrheit streckenweise zugunsten von Nachverhandlungen verhindern konnten, da viele Abgeordnete nicht zu den Sitzungen kamen bzw. bereits ausgewandert oder mit anderen (vorwiegend wirtschaftlichen) Tätigkeiten beschäftigt waren. Unklar war lange, ob überhaupt eine Verfassung verabschiedet werden konnte. Die Autoren hatten u.a. mit der Problematik zu kämpfen, daß nicht das gesamte Territorium des georgischen Staates unter dem Einflußbereich der Politiker in Tbilissi stand: Nach der Niederlage im Bürgerkrieg gegen Abchasien war und ist dieses Teilstück bis heute abtrünnig. Vielfach wurde deshalb gefordert, nur ein Wahlgesetz oder eine "kleine Verfassung" zu verabschieden. Eine Anzahl von Abgeordneten hatte nicht zuletzt aus Anhänglichkeit an ihr eigenes Mandat kein Interesse, die neue Verfassung schnell auf den Weg zu bringen. Die Verabschiedung der Verfassung ist vor allem der Integrationsfigur Schewardnadse zu verdanken, dem es gelang, das Parlament zu disziplinieren und die Verfassungsgebung entschlossen voranzutreiben. Nicht weniger wichtig war dabei auch die politische Einsicht und Weitsicht Chmaladses, der begründete Einwände zugunsten von mehr checks and balances zurückstellte, um - oft unter vier Augen - mit Schewardnadse zu einem Kompromiß in den Formulierungen zu kommen. the State Constitutional Commission, 29. Mai 1995. 


\section{B. Die geltende georgische Verfassung}

\section{Die Verfassung als Provisorium}

Es gibt verschiedene Beispiele provisorischer Verfassungen. Eines war die Bundesrepublik Deutschland. Georgien hat versucht, eine Stabilisierung über Art. 2 Abs. $3 \mathrm{GV}^{14}$ zu erreichen. Die Vorschrift sieht vor, daß der territoriale Staatsaufbau von Georgien "nach der Wiederherstellung der georgischen Staatsgewalt ${ }^{15}$ auf dem gesamten Territorium Georgiens durch ein Verfassungsgesetz nach dem Prinzip der Gewaltenteilung" bestimmt wird. Dabei läßt die Formulierung mehrere Wünsche offen: Der Terminus "Verfassungsgesetz" im Art. 2 Abs. $3 \mathrm{GV}$ ist ein Redaktionsversehen. ${ }^{16}$ Eigentlich müßte hier die Bezeichnung "Organgesetz" stehen. Ein Organgesetz steht von der Hierarchie her zwischen der Verfassung und einem Parlamentsgesetz. Gemäß Art. 66 Abs. 2 GV bedarf es der Mitgliedermehrheit des Parlaments, um es zu verabschieden.

Der Präsident des Verfassungsgerichts bemängelt darüber hinaus, daß der Absatz zu unbestimmt gefaßt sei. Es sei unklar, wer bestimme, wann die Wiederherstellung der georgischen Staatsgewalt eingetreten ist. Dies erzeuge ein Machtvakuum in Hinblick auf die Kompetenzverteilung zwischen dem Zentrum und den Regionen. ${ }^{17}$ Davon wird im letzten Abschnitt die Rede sein.

\section{Der Aufbau der Verfassung}

Die neue Verfassung gliedert sich nach der Präambel in neun Teile: Allgemeine Bestimmungen (Teil 1), Staatsangehörigkeit von Georgien. Die Grundrechte und Freiheiten des Menschen (Teil 2), das Parlament von Georgien (Teil 3), der Präsident von Georgien (Teil 4), das Gerichtswesen (Teil 5), Staatsfinanzen und Kontrolle (Teil 6), Staatssicherheit (Teil 7), Änderung der Verfassung (Teil 8) und die Übergangsbestimmungen (Teil 9).

\section{Die Machtverteilung}

Die geltende Verfassung ist ein Präsidialsystem mit Kabinett, jedoch ohne Premierminister. Diese Konstellation findet man unter den neuen Regierungssystemen der ehemaligen

14

15

16

17

GV = Georgische Verfassung vom 24. August 1995.

Der verwendete Begriff „Jurisdiktion“ meint nicht Gerichtsbarkeit, sondern knüpft an den entsprechenden Begriff im anglo-amerikanischen Rechtskreis an.

Chmaladse, persönliches Interview vom 4. Juli 1996.

Demetraschwili, persönliches Interview vom 17 August 1996. 
Sowjetrepubliken nur noch in Turkmenistan. ${ }^{18}$ Die georgische Verfassung kennt auch keinen Vizepräsidenten. Im Falle der Verhinderung des Präsidenten nimmt der Parlamentsvorsitzende seine Befugnisse wahr (Art. 76 Abs. $1 \mathrm{GV}$ ). Trotz des vorherrschenden Präsidialsystems ist die Macht des Präsidenten durch das Parlament vergleichsweise eingeschränkt. Hinsichtlich der Machtverteilung ergeben sich bei genauerer Analyse der georgischen Verfassung einige Besonderheiten.

\section{Staatsminister und Staatskanzlei}

Art. 18 Abs. 3 GV bestimmt, daß auch der ,Staatsminister ' Mitglied der Regierung ist, "die Staatskanzlei leitet und mit Vollmacht des Präsidenten dessen konkrete Aufträge ausführt". Hinter dem eher unscheinbar formulierten Titel verbirgt sich ein einflußreiches Staatsorgan, das von seinen Funktionen her denen eines Ministerpräsidenten gleichkommt.

Wie wichtig die Funktionen des Staatsministers sind, verdeutlicht die Tatsache, daß eine der ersten Verordnungen, welche nach der Verfassung erlassen wurde, seinen Tätigkeitsbereich regelt. Darin heißt es unter anderem, der Staatsminister kontrolliere, wie die örtlichen Organe Gesetze ausführen ${ }^{19}$, oder, daß er im Rahmen seiner Kanzlei Verträge mit ausländischen Staaten sowie internationalen Organisationen aushandeln und schließen kann.

Die Staatskanzlei ist umfangreich konzipiert. So befinden sich parallele Strukturen der einzelnen Ministerien im Präsidialapparat ${ }^{20}$. Der Präsident hat einflußreiche Funktionsträger in seinem Apparat versammelt, um seine Machtposition zu stärken. So geht z.B. jeder Gesetzesvorschlag, der im Justizministerium erarbeitet wurde ${ }^{21}$, erst über den Tisch des "Parlamentarischen Sekretärs des Präsidenten von Georgien" in der Staatskanzlei, der hier entscheidende Änderungen festlegen kann.

Der Wunsch nach einem Staatsminister kam von Schewardnadse. Er könne doch nicht die ganze Zeit das Kabinett leiten, da müsse man Tätigkeiten koordinieren. Das müsse einer der

18

Vgl. die Aufstellung bei Georg Brunner, Präsident, Regierung und Parlament. Machtverteilung zwischen Exekutive und Legislative, in Otto Luchterhandt (Hrsg.), Neue Regierungssysteme in Osteuropa und der GUS: Probleme der Ausbildung stabiler Machtinstitutionen, S. 113.

19

20

21 
Minister machen ${ }^{22}$. Die Kabinettsleitung konnte er jedoch nicht auf den Staatsminister abwälzen: Zweimal, 1996 und 1998, versuchte Schewardnadse die Rolle des Staatsministers dahingehend auf zuwerten, scheiterte jedoch am Parlament.

Insgesamt wird deutlich, welchen Stellenwert der Präsident den Ministern zuordnet: Das Kabinett wird zum Konsultativorgan degradiert. Dem Präsidenten wird deshalb auch vorgeworfen, daß er durch unterschiedliche Bevorzugung von Mitarbeitern in der Staatskanzlei einerseits und Ministern andererseits, das Prinzip des "divide et impera" gekonnt praktiziere.

\section{Der Nationale Sicherheitsrat}

Die Rolle eines Entscheidungsgremiums auf höchster Ebene übernimmt im georgischen Staat eher der Nationale Sicherheitsrat. Dazu heißt es im Art. 99 Abs. 2 GV: "Zum Zweck der Organisation des militärischen Aufbaus und der Sicherheit des Landes wird der nationale Sicherheitsrat gebildet, den der Präsident von Georgien leitet." Die weitere Besetzung wird im Staatsorganisationsgesetz geregelt. Danach gehören dem Gremium von Amts wegen der Staatsminister, der Außenminister, der Verteidigungsminister, der Minister für Sicherheit, der Innenminister und der Sekretär des Nationalen Sicherheitsrates an. Zusätzlich kann der Präsident noch eine unbestimmte Anzahl von Mitgliedern ernennen. ${ }^{23}$

Anders als im Gesetz über die staatliche Macht von 1992 (vgl. Art. 31 Abs. 3) ist der Nationale Sicherheitsrat dem Parlament gegenüber nicht rechenschaftspflichtig. Vielmehr steht er außerhalb jeder organschaftlichen Kontrolle (außer der des Verfassungsgerichtes). Wie aus informierten Kreisen zu vernehmen ist, berät er aber über einen erweiterten Kreis von Fragen als welche ihm eigentlich übertragen sind. So entschied er 1996 beispielsweise darüber, ob nun das Justizministerium oder die Staatsanwaltschaft über einen Auslieferungsantrag eines fremden Staates entscheiden solle. Eine Frage, die nicht die Sicherheit des Landes betrifft, sondern Kompetenzen der einzelnen Staatsorgane berührt.

\section{Machtverteilung zwischen Präsident und Parlament}

Das Parlament kann die Macht des Präsidenten in verschiedenen Konstellationen begrenzen:

22 Chmaladse, persönliches Interview am 8. August 1996.

23 Art. 4 des Gesetzes über den Nationalen Sicherheitsrat vom 24. Januar 1996. 


\subsection{Kabinettsbildung}

Das Parlament kann einen vom Präsidenten vorgeschlagenen Minister ablehnen oder nicht bestätigen. Daraufhin darf der Präsident denselben Kandidaten nur noch einmal vorschlagen (Art. 77 Abs. 2 GV). Art. 77 Abs. 1 GV bestimmt, daß die Bestätigung einer Regierungsmannschaft durch das Parlament en bloc erfolgen kann. Die einzelnen designierten Mitglieder werden jedoch von den zuständigen Komitees geprüft. Ein Mißtrauensvotum ist nicht vorgesehen.

\subsection{Gesetzgebungsverfahren}

Die Gesetzgebungsinitiative ist weit gefächert: Nicht nur der Präsident, jeder Abgeordnete und die obersten Vertretungsorgane Abchasiens und Adjariens, sondern auch eine Gruppe von mindestens 30.000 Wählern besitzt das Initiativrecht (Art. 67 Abs. $1 \mathrm{GV}$ ).

Nimmt das Parlament einen Gesetzesvorschlag an, so kann der Präsident diesen binnen zehn Tagen mit begründeten Einwänden versehen an das Parlament zurückweisen. Lehnt das Parlament die Einwände des Präsidenten ab und will an der ursprünglichen Version festhalten, so bedarf es dazu gemäß Art. 68 Abs. 4 GV einer dreifünftel Mitgliedermehrheit

des Parlaments. ${ }^{24}$ Verkündet der Präsident das Gesetz daraufhin nicht, so unterschreibt und verkündet es der Vorsitzende des Parlaments (Art. 68 Abs. 5 GV).

\subsection{Haushaltsentwurf}

Nur der Präsident kann dem Parlament einen Haushaltsentwurf vorlegen (Art. 93 Abs. 1 GV). Angesichts der damaligen Blockade zwischen dem US-Repräsentantenhaus und dem US-Präsidenten entschied man, daß der alte Haushalt auch im neuen Haushaltsjahr Bestand hat, wenn das Parlament den Haushaltsentwurf nicht vor Beginn des neuen Haushaltsjahres verabschiedet. Der Präsident kann allerdings einer Änderung seines Entwurfes zustimmen (Art. 93 Abs. 3 und 4 GV).

\subsection{Amtsenthebung}

Eine Amtsenthebung des Präsidenten durch eine Zweidrittelmehrheit des Parlament ist nur möglich, nachdem zuvor das Oberste Gericht eine Straftat gegen den Staat oder das Verfassungsgericht eine Verfassungsverletzung durch den Präsidenten auf Antrag eines Drittels des Parlaments gutachterlich festgestellt hat (Artt. 63 und 75 Abs. 2 GV).

Dies läßt Feldbrugge außer acht. Vgl. Feldbrugge, The Law of the Republic of Georgia, in Rev. CEE Law, Vol. 18 (1992), S. $367-378$. 


\subsection{Ausnahmezustand}

Verhängt der Präsident den Ausnahmezustand, so muß diese Entscheidung binnen 48 Stunden vom Parlament bestätigt werden (Art. 73 Abs. 1 lit. h GV). Nur dann kann der Präsident Dekrete mit Gesetzeskraft erlassen und sogar Grundrechte einschränken (Art. 46 GV), unter anderem das Versammlungsrecht (Art. 25 GV), die Pressefreiheit (Art. 26 Abs. $2 \mathrm{GV}$ ). Besonders besorgniserregend ist die Möglichkeit, das Freiheitsrecht des Art. 18 GV hinsichtlich Verhaftung, Untersuchungshaft und Freiheitsentzug im Ausnahmezustand vollständig aufheben (Art. $46 \mathrm{GV}$ ).

\subsection{Kohabitation}

Die Position des Parlamentes wird im übrigen dadurch gestärkt, daß - anders als noch im "Chicago-Entwurf" - die georgische Verfassung keine Auflösung des Parlaments durch den Präsidenten vorsieht. Andererseits können weder der Staatsminister noch die übrigen Minister mittels eines (konstruktiven) Mißtrauensvotums gestürzt werden.

So ist im bestehenden Modell der georgischen Verfassung auch eine Kohabitation denkbar. Anders als in der US-amerikanischen und der französischen Verfassung hat der Präsident in Georgien aber auch das Recht auf Gesetzesinitiative. Inwieweit sich die georgische Verfassung bei unterschiedlicher Couleur von Parlamentsmehrheit und Präsident als funktionsfähig erweist, wird die Praxis zeigen. Vorteilhaft ist sicherlich, daß die präsidialen und parlamentarischen Vollmachten dezidiert voneinander abgetrennt und festgelegt sind.

\section{Referendum und Plebiszit}

Die Möglichkeit eines Referendums mit Initiativrecht des Präsidenten war in Chicago stark umstritten. Im Widerstreit mit dem Parlament ist der Volksentscheid eine Waffe in der Hand des Präsidenten: Am Parlament vorbei kann er mit Hilfe der Bevölkerung Recht schaffen. Insbesondere in einem Staat, der gerade erst die Spielregeln der Demokratie erlernt, kann ein charismatischer Präsident hier deutlich Einfluß nehmen. Das Referendum aus eigener Initiative des Präsidenten war deshalb im "Chicago-Entwurf" nicht vorgesehen; es wurde nachträglich eingearbeitet. Im Parlament wurde es dann jedoch noch mit einschränkenden Zusätzen versehen. So ist es gemäß Art. 74 Abs. 2 GV unzulässig, eine "Volksbefragung mit dem Ziel der Verabschiedung oder Außerkraftsetzung eines Gesetzes (...) über die Ratifizierung und Kündigung völkerrechtlicher Verträge und Abkommen sowie über Fragen, die die verfassungsmäßigen Grundrechte und Freiheiten anderer Menschen einschränken", durchzuführen. 
Allerdings hat Schewardnadse neben dem Referendum mit dem "Plebiszit" ein weiteres Element direkter Demokratie nachträglich eingeführt. Das Plebiszit hat nur empfehlenden Charakter für die Organe der Staatsgewalt. ${ }^{25}$ Es ist eine allgemeine Bevölkerungsbefragung durch Abstimmung über besonders wichtige Staatsfragen zur Erhellung der Meinung der Wähler Georgiens oder Teilen davon ${ }^{26}$.

Das mit heißer Nadel gestrickte Gesetz diente jedoch nicht so sehr der Erweiterung der Rechte des Präsidenten, sondern vielmehr als Antwort auf die Wahlen in Abchasien. So mußten die Bürger Georgiens am 23. November 1996 ihren ganzen juristischen Sachverstand bemühen, um über folgende wichtige Staatsfrage zu urteilen: "Sind die Wahlen des separatistischen Systems legitimiert oder nicht?" (Mit dem separatistischen System waren die Machthaber in Abchasien gemeint.)

\section{Verfassungsgericht}

Je drei Mitglieder des Verfassungsgerichts werden vom Parlament, dem Präsidenten und dem Obersten Gericht bestimmt (Art. 88 Abs. 2 GV). Das Verfassungsgericht entscheidet unter anderem über Organstreitigkeiten (Art. 89 Abs. 1 lit. b GV), abstrakte Normenkontrollklagen (Art. 89 Abs. 1 lit a GV) und über konkrete Normenkontrolle (Art. 19 Abs. 1 Verfassungsgerichtsgesetz). Die Verfassungsbeschwerde setzt einen normativen Akt voraus, der sich auf Grundrechte aus dem 2. Teil der Verfassung bezieht (Art. 89 Abs. 1 lit $\mathrm{f}$ GV).

Die Möglichkeit einer Verfassungsbeschwerde ist also erheblich eingeschränkt. Staatliche Einzelakte können mit ihr nicht angegriffen werden. Der Bürger wird mit seinem Einzelfall nur dann gehört, wenn der zugrundeliegende Rechtsakt eine Vielzahl von Menschen beschwert. Dabei war eine Ursache für die Limitierung, daß man die Rolle der allgemeinen Gerichte stärken wollte. Die Befürchtung, das Verfassungsgericht würde mit Verfassungsbeschwerden überhäuft werden, stand dagegen eher im Hintergrund.

\section{Die territoriale Gliederung}

Gemäß Art. 4 GV stellt ein Senat die zweite Kammer des Parlament. Der Senat soll nach der Erschaffung "angemessener Bedingungen und nach Bildung der regionalen Verwaltungskörperschaften auf dem gesamten Territorium von Georgien" gebildet werden. Aus

Art. 8“ Ziff. 4 des Gesetzes über das Referendum vom 29. Oktober 1996.

26

Art. 8‘ Ziff. 1 des Gesetzes über das Referendum vom 29. Oktober 1996. 
der Verfassung ist allerdings nicht ersichtlich, welche Funktionen, außer konsultativen, er übernehmen sollte.

Grundsätzlich sind Angelegenheiten von regionaler Bedeutung auf der Basis der Selbstverwaltung zu regeln (Art. 2 Abs. $4 \mathrm{GV}$ ). Das ist bislang nur unter Einschränkungen möglich: Im Regelfall wählt das lokale Parlament, das "Sakrebulo" genannt wird, einen Bürgermeister. Dieser hat umfangreiche Kompetenzen, während der Sakrebulo hauptsächlich über das Budget entscheidet.

Anders ist dies jedoch bei den sechs größten Städten des Landes, in denen auch der Großteil der georgischen Bevölkerung lebt. Hier werden die Bürgermeister direkt vom Präsidenten ernannt. Gleichzeitig kann der Präsident auch den Sakrebulo auflösen, wenn das lokale Parlament sich nicht auf ein Budget einigen kann. Dies ist eine Kompetenz, die im Widerspruch zu Art. 73 Abs. 1 lit. i GV steht. Danach darf der Präsident nämlich nur mit Zustimmung des Parlaments die Tätigkeit anderer Vertretungsorgane aufheben, wenn "die Souveränität des Landes, die territoriale Integrität und die Ausübung der Befugnisse der verfassungsmäßigen staatlichen Machtorgane beeinträchtigt wird".

Eine Koalition oppositioneller Parteien wollte deshalb im September 1997 ein Referendum mit der Frage: "Sind sie damit einverstanden, daß der Vorsitzende der Exekutive aller Lokalen Gewalten (...) gewählt werden soll?'" initiieren. Nach Verbot dieser Initiative entschied das erstinstanzlich zuständige Oberste Gericht, das avisierte Referendum versuche Gesetzgebung via Volksbefragung zu verabschieden und verstoße damit gegen den oben zitierten Art. 74 Abs. 2 GV. Dabei handelte es sich jedoch um eine Verfassungsfrage, die höchstens über eine konkrete Normenkontrolle vom Verfassungsgericht hätte entschieden werden können. ${ }^{2}$

\section{Fazit}

Die Republik Georgien hat sich inmitten einer schwierigen Übergangsphase eine neue Verfassung gegeben, die dem jungen Staat Stabilität verleiht. Im Gegensatz zu vielen anderen Transformationsstaaten hat sich Georgien für ein weniger autokratisches System entschieden. Während die Kompetenzen zwischen Präsident und Parlament weitestgehend definiert sind, geben die starke Stellung des Staatsministers samt Staatskanzlei sowie die unkontrollierte Machtfülle des Nationalen Sicherheitsrates ebenso Anlaß zu Bedenken wie die Eingriffsbefugnisse des Präsidenten in lokale Entscheidungsstukturen. 
Die transkulturelle Rechtsberatung hat der Verfassungsgebung in Georgien entscheidende Impulse gegeben und zwar sowohl hinsichtlich der Formulierung von Artikeln und der Konstruktion eines ausgewogenen und praktikablen Staatsgefüges als auch in der Fähigkeit, die unterschiedlichen Entwürfe zu bündeln und Kompromißähigkeit zu schaffen. Leider erfolgte nur eine geringe Nachbetreuung. Ob diese allerdings ein Festhalten an den Lösungen des "Chicago-Entwurfs" bewirkt hätte, darf bezweifelt werden. Georgien befand sich im politischen, rechtlichen, wirtschaftlichen und sozialen Umbruch. Wie vehement um die Kompetenzen der Staatsorgane gestritten wurde, gibt Aufschluß darüber, welche gegensätzlichen Interessensphären aufeinanderprallten. Optimistisch stimmt dagegen, daß Georgien dennoch zu einer praktikablen Verfassung gefunden hat, die hoffentlich noch lange Jahre ihre Tauglichkeit unter Beweis stellen wird. 


\title{
The Protection of Individual Rights in the Constitution of Colombia
}

\author{
By Andreas Timmermann
}

The starting point of this contribution is that the Colombian constitution reflects both the social and economical requests of a developing country and the most important principles of European constitutional law. The resulting compromise leads to a characteristic systematization and differentiation of individual rights. As a consequence the constitutional court handles the protection of individual rights rather flexibly. The court develops special guiding principles and accommodates European doctrines of fundamental rights to the social reality in Colombia.

\section{New Constitutional Structures in Georgia}

\section{By Wolfgang Gaul}

Over the last decade, the Republic of Georgia has been at the forefront of political changes within the Community of Independent States (CIS). What type of governmental structure should a country choose that has undergone such severe political changes? It needs to find a way to comfort the people's desire for security and stabilisation but should also avoid establishing authoritarian structures.

The current Georgian Constitution establishes a notable balance between President and Parliament. However, the vague responsibilities of the State Minister and the National Security Council tip the scale towards presidential powers. The article reveals which different approaches had been proposed during the constitution-making process and which constitutional archetypes influenced the key players of the country.

Transcultural legal consultation supported and escorted the different existing drafts. A compromise was elaborated during an international conference in Chicago. However, it all ended differently, when Shevardnadze and his Minister of Justice, Ninidze, turned the tide towards stronger presidential powers.

Will these powers be sufficient to unify a country which is on the verge of separation primarily due to ethnic differences or instead, should its regions and local authorities be granted more autonomy? The rules of the game are all laid out in the constitution - and the game itself is called politics. 